\title{
Circuit
}

Musiques contemporaines

\section{La construction du champ identitaire de la musique actuelle en Amérique du Nord : enquête sur la filiation avec la Semaine internationale de musique actuelle Shaping the identity of "Today's Music" in North America: influence of the International Week of Today's Music}

\section{Sophie Stévance}

Volume 21, numéro 3, 2011

Musique automatiste ? Pierre Mercure et le Refus global

URI : https://id.erudit.org/iderudit/1006361ar

DOI : https://doi.org/10.7202/1006361ar

Aller au sommaire du numéro

Éditeur(s)

Les Presses de l’Université de Montréal

ISSN

1183-1693 (imprimé)

1488-9692 (numérique)

Découvrir la revue

Citer cet article

Stévance, S. (2011). La construction du champ identitaire de la musique actuelle en Amérique du Nord : enquête sur la filiation avec la Semaine internationale de musique actuelle. Circuit, 21(3), 75-86.

https://doi.org/10.7202/1006361ar
Résumé de l'article

En quoi le courant de la musique actuelle, tel que connu depuis 1979 avec la fondation des Productions SuperMémé, puis des Productions SuperMusique, est-il affilié à la Semaine internationale de musique actuelle (SIMA) organisée par Pierre Mercure, à Montréal, en 1961 ? L’article tente d'y répondre en questionnant les fondements de l'expression « musique actuelle " pour faire ressortir les éléments historiques qui tracent puis jalonnent ses significations depuis ses premières utilisations. L'auteure montre que le mouvement " actualiste " partage avec l'événement de Mercure, par lequel le compositeur souhaitait renouveler l'acte créateur par une programmation non conventionnelle s'opposant à la « vieille garde », bien plus qu'un nom : il en porte et réalise peut-être la visée esthétique et sociale en s'opposant à certaines zones des pôles culturels par des méthodes de création telles que l'improvisation libre ou la dynamique collective. Mais au-delà de ce projet, c'est bien dans la lignée de Mercure et des événements musicaux de la SIMA que les actualistes ont puisé leur inspiration et construit leur champ identitaire. 


\section{La construction du champ identitaire de la musique actuelle en Amérique du Nord: enquête sur la filiation avec la Semaine internationale de musique actuelle}

Sophie Stévance

La Semaine internationale de musique actuelle de Montréal (SIMA) organisée en 1961 par Pierre Mercure dans le cadre des Festivals de Montréal est un événement majeur : il a permis de faire entendre, aux côtés des Garant, Stockhausen, Ligeti, Schaeffer, Xenakis ou Varèse, la musique expérimentale «du temps présent», aussi provocante et hétéroclite soit-elle, avec notamment la création d'Atlas Eclipticalis de Cage, du Piano Concert for David Tudor de Richard Maxfield, ou encore d'œuvres de Mauricio Kagel, de Christian Wolff et d'Earle Brown. Pour comprendre ce que l'on entend par «musique expérimentale», il faut se référer aux réflexions de Cage exposées dans «Experimental music : Doctrine ${ }^{»}$ (1955). Référer aux conceptions cagiennes n'est pas casuel étant donné la place privilégiée que Mercure accordait à l'École de New York².

La musique expérimentale d'un Stockhausen ou d'un Xenakis n'est pas celle de Cage alors mise à l'honneur par Mercure pour la SIMA. Il y a une très nette distinction entre ces deux esthétiques, distinction par ailleurs corroborée par Marie-Thérèse Lefebvre lors de nos entretiens :
1. Cage, 1961, p. 13-17.

2. Voir les commentaires suscités par la programmation de Mercure priorisant Cage et ses consorts américains (Vallerand, 1961, p. 8) ou ceux d'Eric McLean (1961, p. 10). 
3. Marie-Thérèse Lefebvre, entretien avec Sophie Stévance, 5 novembre 2011.

4. Pépin, 1961, p. 29-31. Notre référence à l'expression "nouvel art" lancée par Pépin, compositeur réputé conservateur, n'est pas qu'anecdotique : elle est de nature à marquer les réactions controversées suscitées par la programmation "tournée vers le nouveau monde" de ce festival, et se place au centre d'un champ de forces entre conservatisme (ou prolongement de la tradition classique) et volonté de tourner le dos à la tradition européenne.

5. Gervais, 1990, p. 7.
Le mot expérimental ne s'adresse pas à l'avant-garde française. L'expérimental se rapporte à ce que l'on appelle l'américanité, qui est ce désir, dans ce nouveau monde, d'expérimenter. Cela remonte à Charles Ives et Henry Cowell, ainsi qu’à Marcel Duchamp. C'est un courant qui, pour moi, est en réaction [...]. Je le retrouve en 1961 par la volonté de Mercure d'affirmer notre appartenance à l'Amérique, à ce nouveau monde qui est le territoire de l'expérimentation, de la découverte. C'est donc en ce sens que le mot «expérimental» s'applique à la musique américaine, moins à la musique européenne, dont les attitudes, aussi nouvelles soient-elles, s'inscrivent dans la continuité rationnelle de la tradition européenne 3 .

La volonté de Mercure était de défendre une esthétique expérimentale américaine, différente des expérimentations de l'école européenne. Notre hypothèse est que la musique actuelle, qui correspond depuis 1979 à un courant musical à part entière, prend son souffle dans les directions esthétiques ; elle pourrait même correspondre à ce «nouvel art » généré par ce festival de controverses et d'esthétiques.

\section{Un projet global}

Depuis les années 1980, plus particulièrement au Québec, le terme musique actuelle désigne un mouvement musical d'avant-garde dont les musiciens, en réaction contre les institutions, en déconstruisent les valeurs en leur opposant ce que celles-ci ont mis à l'opprobre : pêle-mêle bruitisme, sons électroniques, improvisation libre, rock, musique du folklore ou jazz. Cette influence du jazz a été relevée par Raymond Gervais : "ce sont des musiciens francophones (comme ceux associés à [l'étiquette de disque] Ambiances Magnétiques) qui ont le plus radicalement remis en cause le modèle américain de jazz pour lui substituer un modèle québécois en devenir (fidèle à cet égard à l'esprit même du jazz d'origine qui se voulait une musique d'émancipation, d'autonomie) 5 ». Ces propos sont toutefois nuancés par Jean Derome (son trio Derome-Guilbeault-Tanguay témoigne d'un riche savoir-faire du jazz d'origine) ou par Danielle Palardy Roger, qui nous affirment chercher à repousser les frontières de la création sans viser le jazz américain particulièrement, mais l'ensemble des académismes. L’œuvre actualiste représente donc une création spontanée où s'établissent de nouvelles relations entre les musiciens sur scène et le public, relations d'autant plus marquées par l'absence de hiérarchie entre les compositeurs et les musiciens, ainsi que par la participation des compositeurs en tant qu'interprètes de leur propre musique. Gérant sa propre production, ses membres œuvrent sans se soucier ni des modes en vigueur ni des conventions et se font fort de mettre constamment en lumière la participation des femmes à la recherche musicale. Le mouvement constitue ainsi une structure parfaitement organisée que l'on peut saisir en observant 
les stratégies esthétiques mises en place par Mercure. Le compositeur ne souhaitait-il pas déconstruire, au sens derridien du terme, les conventions musicales d'alors en leur opposant ce que celles-ci avaient jusqu'ici exclu? Lors de la table ronde animée par Jean-Marie Beaudet en 1961, avec Maryvonne Kendergi, Pierre Mercure, Eric McLean et Jean Vallerand, succédant au festival, Pierre Mercure donne une définition de ce qu'il entend par «musique actuelle» à cette époque :

Par musique actuelle, j'entends cette nouvelle façon d'approcher la musique, la recherche du son $[\ldots]$, une importance du son supérieure à la forme, à la mélodie et à des correspondances avec des formes établies par les siècles précédents. La musique de Bali, par exemple, s'écoute sans savoir s'il s'agit d'une forme ou d'une autre, si c'est une danse ou pas; la musique hindoue s'entend avec beaucoup d'intérêt même si on ne connaît pas le raga qui lui a donné naissance. Dans ces musiqueslà, le son nous convainc. Dans la musique concrète [de Pierre Schaeffer], le son devrait nous convaincre mais ne nous convainc pas parce que l'on est victime d'un ensemble, d'un fait social qui nous amène toujours à la même sorte de musique, au même concert, etc. Je pense que c'est une question d'ordre psychologique ${ }^{6}$.

Selon Marie-Thérèse Lefebvre, la musique actuelle correspondait également (et surtout), pour Mercure, à «l'intégration du pluridisciplinaire sur scène et du courant américain 7 ». Témoin de l'engagement du compositeur en ce sens : la programmation de musiques accompagnées d'une chorégraphie (par exemple Incandescence de Pierre Mercure avec la chorégraphie de Françoise Riopelle), des projections de cinéma expérimental sur des musiques concrètes ${ }^{8}$, ou encore de la présentation d'œuvres de compositeurs qui allaient bientôt former le groupe Fluxus (Yoko Ono, Toshi Ichiyanagi, David Behrman et Richard Maxfield). Tous sont issus de la New School for Social Research, à New York, où ils avaient suivi l'enseignement de John Cage et participé aux premières séries de concerts-performances organisées par La Monte Young dans le loft de Yoko Ono en 1960 et 1961.

Une dimension internationale est aussi prégnante dans ce que Mercure entend par musique actuelle. Ainsi, dans le programme de «La société des Festivals de Montréal » daté du 25 juin 1961, il écrit qu' «afin d'assurer les caractéristiques les plus essentielles de cette première Semaine Internationale de Musique Actuelle, ce programme pourra être modifié jusqu’à la dernière minute, pour faire place à des œuvres susceptibles de mieux représenter l'Actualité ou l'Internationalité de la musique d'aujourd'hui 9 ». Fort de ces trois principes de base - «Actualité, Internationalité et Interpénétration des Arts Visuels» -, Mercure voulait également offrir «au spectateur de mieux communiquer à l'art musical d'aujourd'hui ${ }^{10}$ ». Il s'agissait donc de créer de nouvelles conditions d'écoute à la lumière de celles déjà en vigueur dans les
6. Je transcris. Pierre Mercure, Festival du mercredi (Musique actuelle 1961), disque à 8'10. Table ronde animée par Jean-Marie Beaudet, avec Maryvonne Kendergi, Pierre Mercure, Eric McLean et Jean Vallerand. Archives de MarieThérèse Lefebvre. Plus loin (à 15'35), Mercure précise que la musique actuelle est représentée par la musique concrète, la musique électronique et la musique aléatoire de Cage. [ndlr] Voir aussi la rubrique Document.

7. Marie-Thérèse Lefebvre, entretien avec Sophie Stévance, 5 novembre 2010

8. Par exemple Les Objets inanimés de Briscot et Schaeffer (1960), Point et contrepoint de Cioni Carpi (1961), Conte de Kameler et Mâche (1960).

9. "Note importante", fonds Pierre Mercure des Archives nationales du Québec, Programme 06-M, P9o/1-16.3, 61/08/03 au 08. Original dactylographié, 6 pages.

10. Pierre Mercure, première rencontre du comité de préparation de la SIMA. Document sur les activités (06-M, P9o/2-5.2). 61/05/11, Montréal. Original dactylographié, 5 pages. Fonds Pierre Mercure, Archives nationales du Québec. 
11. Document sur les activités 2-5.5. Montréal, 62/08/og, de Pierre Mercure, pour Robert Letendre, président. Seconde version du projet pour la Société des Festivals de Montréal. Deuxième semaine internationale de musique actuelle, saison d'été 1962. Original dactylographié, 7 pages.

12. Marie-Thérèse Lefebvre, entretien avec Sophie Stévance, 5 novembre 2010.

13. Côté, 1995, p. 27-29.
14. Ce que note d'ailleurs Dyane Raymond dans son ouvrage consacré à Derome : ainsi l'actualiste est-il "heureux d'appartenir à une communauté qui exprime son identité et son appartenance à la famille, à la société et au pays, par la musique actuelle" (Raymond, 2007, p. 23).

15. Je transcris. Pierre Mercure, Festival du mercredi (Musique actuelle 1961), disque à 17'20. Table ronde animée par Jean-Marie Beaudet, avec Maryvonne Kendergi, Pierre Mercure, Eric McLean et Jean Vallerand. Archives de MarieThérèse Lefebvre.

16. Danielle Palardy Roger, entretien autres arts, et ainsi «démontrer que la musique d'aujourd'hui est en train de subir une évolution non différente de celle déjà opérée en peinture, en sculpture et en cinéma contemporains ${ }^{11}{ }^{\prime}$. La réception figurait donc elle aussi dans le projet global de Mercure.

\section{Les enjeux d'un qualificatif}

La musique actuelle selon Mercure était incarnée par des compositeurs de l'avant-garde américaine et européenne : le courant actualiste prend ainsi sa source au centre d'un champ de forces dont la structure jaillit de l'opposition entre deux pôles culturels, que Marie-Thérèse Lefebvre définit aujourd'hui ainsi : «Ces mots définissent une activité dans le temps. On est contemporain ou on est actuel. Très rapidement, ces mots sont devenus des positions esthétiques, avec tout ce que cela implique en termes de choix de répertoires ${ }^{12}{ }^{»}$. Cette dénomination implique donc certains enjeux. L'un des principaux réside dans la force d'identification du courant actualiste qui, dans la chronologie musicale du Québec, s'inscrit dans la lignée de la SIMA.

L'expression musique actuelle peut dérouter par la limitation temporelle qu'elle semble imposer. Se prêtant tout à la fois à l'exercice et refusant le jeu du «cirque nominatif ${ }^{13}$ », les actualistes ont contesté l'essai définitoire. On relève pourtant, au fil de nos recherches et entretiens, que des actualistes comme Jean Derome, Joane Hétu ou Danielle Palardy Roger y recourent : ils montrent une volonté identitaire qui préside à l'entreprise de définition, justifiant ainsi le plein emploi de l'expression musique actuelle pour les désigner et la nécessité de comprendre ce qu'elle recouvre. L'objet est de saisir l'identité musicale que les actualistes revendiquent à travers elle ${ }^{14}$, et par là même d'envisager sa capacité à incarner cette «nouvelle conception sonore qui soit l'égale de notre sensibilité qui a évolué 15 », projetée par Mercure. Palardy Roger témoigne de cette filiation avec Mercure et la SIMA, même si cette parenté est indirecte étant donné l'évolution du terme et de ses utilisations au fil des décennies. Entre 1961 (SIMA) et 1979 (date du regroupement des actualistes), on assiste à un long processus de reconnaissance. Les actualistes ont pris connaissance de la SIMA en 1990 alors qu'ils participaient au Montréal musiques actuelles (1-11 novembre 1990) organisé par Jean Piché dans la tradition du New Music America : «En 1990, nous avons traversé une période de remise en question, concernant les directions que l'on souhaitait donner à notre groupe. On a alors pris conscience que notre musique actuelle prenait ses racines dans l'événement de Mercure. Cela ne faisait aucun doute. Ce fut une révélation... C'est comme si on avait retrouvé un parent perdu, un aïeul... ${ }^{16}$ » 
Pour saisir cette identité musicale, il faut alors tisser les liens historiques qui mènent de la SIMA, de la signification du terme «musique actuelle » au cours de ses différents emplois depuis 1961, jusqu'au courant actualiste tel que nous l'entendons depuis les années 1980.

Il nous faut à présent décrire le trajet de l'utilisation de l'expression. Nos entretiens avec les actualistes et spécialistes de la musique québécoise ${ }^{17}$ font ressortir les étapes suivantes permettant de tracer la chronologie du courant de la musique actuelle à partir de l'événement de Mercure.
17. Nous nous référons, en plus des archives reconstituées (à la suite d'un incendie en 2005) des Productions SuperMusique, à l'importante chronologie de la musique québécoise réalisée par Marie-Thérèse Lefebvre et Jean-Pierre Pinson (2009, p. 299-300, 324-325, 330-331, 333).

ANNEXE 1 Chronologie de la musique actuelle

\begin{tabular}{|c|c|c|}
\hline Dates & Événements & Figures marquantes \\
\hline 1961 & Semaine internationale de musique actuelle de Montréal (SIMA) & Pierre Mercure - John Cage \\
\hline \multirow[t]{2}{*}{1968} & $\begin{array}{l}\text { Fondation de L'Infonie : spectacles multidisciplinaires alliant poésie, } \\
\text { improvisation, arts visuels et chorégraphiques, parfois proches de la performance, } \\
\text { à la recherche de timbres nouveaux et où le public participe à la création }\end{array}$ & Walter Boudreau et Raôul Duguay \\
\hline & Concerts du Quatuor de jazz libre du Québec & $\begin{array}{l}\text { Jean Préfontaine - Yves Charbonneau - } \\
\text { Maurice Richard - Guy Thouin }\end{array}$ \\
\hline \multirow[t]{2}{*}{1975} & Année internationale de la femme & \\
\hline & Parachute, revue d'art contemporain & Chantal Pontbriand \\
\hline 1977 & Fondation du Feminist Improvising Group (FIG) à Londres & Maggie Nicols - Lindsay Cooper \\
\hline \multirow[t]{3}{*}{1978} & Fondation de l'Association de musique actuelle de Québec & Gisèle Ricard \\
\hline & Fondation du groupe Arcanson & Danielle Palardy Roger - Diane Labrosse \\
\hline & Fondation de l'ensemble de musique improvisée de Montréal & $\begin{array}{l}\text { Jean Derome - Robert Leriche - Pierre St- } \\
\text { Jacques - Claude Simard - René Lussier }\end{array}$ \\
\hline 1979 & Fondation du groupe SuperMémé & $\begin{array}{l}\text { Danielle Palardy Roger - Joane Hétu - } \\
\text { Diane Labrosse }\end{array}$ \\
\hline \multirow[t]{2}{*}{1980} & $\begin{array}{l}1^{\text {er }} \text { concert de Wondeur Brass à la Salle Polonaise pour le } 5^{\mathrm{e}} \text { anniversaire } \\
\text { de la Librairie des femmes de Montréal }\end{array}$ & $\begin{array}{l}\text { Danielle Palardy Roger - Joane Hétu - } \\
\text { Diane Labrosse - Gin Bergeron - } \\
\text { Claude Hamel - Geneviève Letarte - } \\
\text { Dyane Raymond - Danielle Broué - } \\
\text { Martine Leclercq }\end{array}$ \\
\hline & Organisation du colloque «Performance : postmodernisme et multidisciplinarité» & Chantal Pontbriand \\
\hline 1983 & 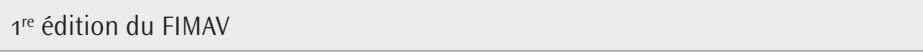 & Michel Levasseur \\
\hline \multirow[t]{5}{*}{1984} & Fondation du groupe Les Granules & Jean Derome - René Lussier \\
\hline & Création du label Ambiances Magnétiques & $\begin{array}{l}\text { Jean Derome - André Duchesne - } \\
\text { René Lussier - Robert M. Lepage }\end{array}$ \\
\hline & $\begin{array}{l}\text { Début de l'émission radiophonique "Musique actuelle", Radio-Canada } \\
\text { (succède à Musique de notre siècle créée en } 1967 \text { et animée par Serge Garant) }\end{array}$ & Hélène Prévost \\
\hline & $\begin{array}{l}1^{\text {er }} \text { concert de Wondeur Brass, } 4 \text { e Congrès international "Femmes et musiques" } \\
\text { à Paris }\end{array}$ & $\begin{array}{l}\text { Danielle Palardy Roger - Joane Hétu - } \\
\text { Diane Labrosse }\end{array}$ \\
\hline & $\begin{array}{l}\text { Rencontre de Wondeur Brass avec le groupe Les Diaboliques constitué } \\
\text { de Joëlle Léandre, Annick Nozati et Irene Schweizer }\end{array}$ & \\
\hline
\end{tabular}




\begin{tabular}{|c|c|c|}
\hline & $\begin{array}{l}\text { Rencontre de Wondeur Brass avec André Duchesnes (qui guide les musiciennes } \\
\text { vers Ambiances Magnétiques) et Chris Cutler du label Recommended Records } \\
\text { (fondé en 1978) }\end{array}$ & \\
\hline 1985 & Sortie du premier 33 tours Ravir de Wonder Brass (label Ambiances Magétiques) & $\begin{array}{l}\text { Danielle Palardy Roger - Joane Hétu - } \\
\text { Diane Labrosse }\end{array}$ \\
\hline 1986 & $\begin{array}{l}\text { Sortie du premier } 33 \text { tours de Les Poules, Les Contes de l'amère loi } \\
\text { (label Ambiances Magétiques) }\end{array}$ & $\begin{array}{l}\text { Danielle Palardy Roger - Joane Hétu - } \\
\text { Diane Labrosse }\end{array}$ \\
\hline 1987 & Fondation du groupe Les Poules parallèlement à Wondeur Brass & $\begin{array}{l}\text { Danielle Palardy Roger - Joane Hétu - } \\
\text { Diane Labrosse }\end{array}$ \\
\hline 1988 & Festival international des musiciennes innovatrices & Productions SuperMusique \\
\hline \multirow[t]{5}{*}{1990} & Festival Montréal musiques actuelles & Jean Piché \\
\hline & $\begin{array}{l}\text { Utilisation récurrente, dans la presse écrite, de l'expression "musique actuelle» } \\
\text { pour précisément désigner le groupe des actualistes }\end{array}$ & $\begin{array}{l}\text { Andrew Jones (Montreal Mirror) - } \\
\text { Lyne Crevier (Le Devoir) - Andrée Laurie } \\
\text { (Le Musicien Québécois), etc. }\end{array}$ \\
\hline & Tournée européenne de Les Granules & Jean Derome - René Lussier \\
\hline & Wondeur Brass devient Justine & $\begin{array}{l}\text { Danielle Palardy Roger - Joane Hétu - } \\
\text { Diane Labrosse - Marie Trudeau }\end{array}$ \\
\hline & $\begin{array}{l}\text { Les labels Ambiances Magnétiques, Productions } \\
\text { SuperMémé-SuperMusique se regoupent et lancent le label DAME } \\
\text { (Distribution Ambiances Magnétiques Etcetera) }\end{array}$ & $\begin{array}{l}\text { Danielle Palardy Roger - Joane Hétu - } \\
\text { Diane Labrosse - Jean Derome - } \\
\text { André Duchesne, René Lussier - } \\
\text { Robert M. Lepage }\end{array}$ \\
\hline 1991 & La revue Circuit consacre le volume $1, \mathrm{n}^{\circ} 2$, au Montréal / musiques actuelle & Jean-Jacques Nattiez - Jean Piché \\
\hline 1992 & Super-Mémé devient SuperMusique & $\begin{array}{l}\text { Danielle Palardy Roger - Joane Hétu - } \\
\text { Diane Labrosse }\end{array}$ \\
\hline \multirow[t]{2}{*}{1993} & $\begin{array}{l}1^{\text {er }} \text { concert "Une théorie des ensembles: Ambiances Magnétiques en concert" - } \\
\text { Productions SuperMémé }\end{array}$ & $\begin{array}{l}\text { Danielle Palardy Roger - Joane Hétu - } \\
\text { Diane Labrosse - Jean Derome - } \\
\text { André Duchesne - René Lussier - } \\
\text { Robert M. Lepage }\end{array}$ \\
\hline & Fondation du groupe Jean Derome et les Dangereux Zhoms & $\begin{array}{l}\text { Jean Derome - Pierre Cartier - } \\
\text { Guillaume Dostaler - René Lussier - } \\
\text { Pierre Tanguay - Tom Walsh }\end{array}$ \\
\hline 1994 & Fondation du groupe Castor et Compagnie & $\begin{array}{l}\text { Joane Hétu - Jean Derome - } \\
\text { Diane Labrosse - Pierre Tanguay }\end{array}$ \\
\hline \multirow[t]{3}{*}{1995} & Début de l'émission radiophonique "Le Navire Night", Radio-Canada & Hélène Prévost - Mario Gauthier \\
\hline & $\begin{array}{l}\text { Publication du livre Plunderphonics, 'Pataphysics \& Pop Mechanics. } \\
\text { An Introduction to musique actuelle (Wembley, SAF Publishing, 1995) }\end{array}$ & Andrew Jones \\
\hline & La revue Circuit consacre le volume $6, \mathrm{n}^{\circ} 2$, à la musique actuelle & $\begin{array}{l}\text { Jean-Jacques Nattiez - Jean Derome - } \\
\text { Danielle Palardy Roger - Joane Hétu - } \\
\text { Michel F. Côté - Raymond Gervais - } \\
\text { Hélène Prévost }\end{array}$ \\
\hline \multirow[t]{2}{*}{1998} & Fondation de l'Ensemble SuperMusique & Productions SuperMusique \\
\hline & $\begin{array}{l}\text { Début des activités Jeune Public des Productions SuperMémé, } \\
\text { "Dans le ventre de la musique actuelle" }\end{array}$ & $\begin{array}{l}\text { Danielle Palardy Roger - Joane Hétu - } \\
\text { Diane Labrosse - Jean Derome - } \\
\text { Pierre Tanguay - Martin Tétreault }\end{array}$ \\
\hline 2000 & Festival SuperMicMac - Hommage à La Bolduc & $\begin{array}{l}\text { Diane Labrosse - Danielle Palardy Roger - } \\
\text { Joane Hétu - Jean René - Lori Freedman - } \\
\text { Lee Pui Ming - Marie Pelletier - } \\
\text { Allison Cameron }\end{array}$ \\
\hline
\end{tabular}




\begin{tabular}{|c|c|c|}
\hline 2001 & Fondation du groupe Nous perçons les oreilles & Jean Derome - Joane Hétu \\
\hline & Fondation de Plinc! Plonc! & Jean Derome - Pierre Tanguay \\
\hline \multirow[t]{2}{*}{2004} & $\begin{array}{l}\text { Thèse de sociologie : "Étude sociologique de la musique actuelle du Québec: } \\
\text { le cas des Productions SuperMusique et du Festival international de musique } \\
\text { actuelle de Victoriaville", Université de Montréal }\end{array}$ & Anne Robineau \\
\hline & 25 ans de SuperMusique... & Productions SuperMusique \\
\hline
\end{tabular}

On mentionnera encore les autres points de rencontre de la musique actuelle à Montréal : la Sala Rossa, les brasseries ou cafés-concerts (Le Cheval Blanc ou l'Usine C), le théâtre La Chapelle, Traquen'Art ou la Casa Obscura. Il faut encore mentionner les autres apports substantiels à la visibilité de la musique actuelle, telles que les revues spécialisées comme Musicworks ou Wire.

\section{L'identité de la musique actuelle}

L'amorce des années 1980 marque donc les débuts de la musique actuelle et de la reconnaissance de ses membres en tant que groupe :

De 1980 à 1985, Wondeur Brass a donc surtout évolué parmi les groupes de femmes et les événements féministes, toutefois rapidement nous désirions être perçues d'abord comme musiciennes et ensuite féministes et non l'inverse. C'est donc à partir de la sortie de Ravir, le premier microsillon de Wondeur Brass que l'on s'est définies comme un groupe de musique actuelle, et ce fut le début d'une autre aventure ${ }^{18}$.

Dans l'édition du 4 mai 1989 du Globe and Mail, Mark Miller commentait un disque produit par Ambiances Magnétiques : «The latest LP from Montreal's east end, like all the rest, must come with the qualification that it's not, ah, exactly, um, jazz [...] musique actuelle, they call it at the corner of St-Denis and Mont-Royal ${ }^{19}{ }^{»}$. Cette remarque laisse croire à une identité musicale spécifique des artistes de la scène actualiste, géographiquement implantée et culturellement structurée.

L'identification est autant une construction sociale qu'une prise de conscience de sa culture. L'actualiste est un individu qui œuvre au sein d'un collectif auprès duquel il se reconnaît des valeurs communes. Cette situation, spécifique aux écoles artistiques, a fait dire que l'improvisation libre «est une musique d'individus où il est nécessaire de compter les uns sur les autres $[. .$.$] . Paradoxalement, c'est une musique qui est totalement indivi-$ dualiste et qui a à voir avec du collectif ${ }^{20}$ ». Aussi cette « conscience » accrue "d'une identité musicale ${ }^{21}$ » vécue par les actualistes incite-t-elle à investiguer ce sentiment partagé par une communauté encore mal comprise. Il ne s'agit là que d'un voile de contradictions logiques à lever, si tant est que la
18. Joane Hétu in Arroyas, 2005, p. 4-9.

19. Cette intersection a été illustrée par Circuit sur la page couverture du numéro consacré au mouvement en 1995.

20. Léandre, 2008, p. 67.

21. Ibid., p. 119 
22. Bourdieu et Wacquant, 1992, p. 76.

23. Voir l'Encyclopédie canadienne, www.thecanadianencyclopedia.com/ index.cfm?PgNm $=$ TCE\&Params $=$ Q1A RTQ0001709 notion d'identité soit elle-même susceptible d'admettre rationnellement des déterminations contraires. Pour comprendre celle de l'actualiste, deux types de référents sont possibles : psychoculturels (système de valeurs culturelles, habitudes collectives, système affectif et cognitif) et psychosociaux (références sociales, compétences reconnues, potentialités du devenir).

Ces déterminants lui assurent son assise théorique et se situent implicitement au sein de l'appellation musique actuelle, dont les enjeux remontent à l'événement de Mercure et sont beaucoup plus considérables que ce que l'on a jadis souhaité y voir : ils permettent d'observer des « effets de champ ${ }^{22}$ » visant à définir le statut spécifique d'un véritable champ artistique.

\section{Référents psychoculturels}

La culture québécoise s'est forgé une reconnaissance internationale solide grâce à ses festivals. Les événements musicaux (que l'on songe au Festival International de Jazz de Montréal ou au FIMAV), dont la mission est de diffuser les musiques expérimentales et d'improvisation les plus nouvelles, font la fierté du milieu musical en Amérique du Nord. Le festival donne la possibilité d'établir des relations culturelles d'ampleur internationale, de soutenir la création musicale au sein même de la discipline et de favoriser son essor en maintenant un équilibre entre innovation et tradition pour conserver une programmation vivante et populaire. Vecteur de libre expression et d'émotions partagées, le festival est également un facteur de cohésion sociale parce qu'il transmet et affirme les valeurs de la communauté. Si l'on tient compte des stratégies de médiatisation qu'elle a su développer, la musique actuelle est motivée par une dynamique démocratique mise à la portée du plus grand nombre. Malgré ses exigences, elle participe à la culture du monde et cherche à se rendre accessible à un public moins initié. Or, le festival, en tant que phénomène de socialisation et de démocratisation de la musique, tend à une modification des rapports entre le créateur et le récepteur où ce dernier est invité à prendre la parole, à réagir à et avec celle de l'artiste en temps réel.

Le terme actuel renvoie précisément à la construction de l'œuvre ici et maintenant, à ce «qui existe, se passe au moment où l'on parle», comme le définit le Petit Robert. Pour cette raison sera pointée la traduction en anglais du titre donné par Mercure - International Week of Today's Music ${ }^{23}$ - parce qu'elle met l'accent sur cette dimension du temps présent (Today). Si l'on tient compte des aspirations artistiques de Mercure et de ses œuvres, il ne fait aucun doute qu'il souhaitait, dès le titre de son festival, aller au-delà de la simple dimension temporelle de la musique qu'il voulait y voir représentée : il désirait s'ouvrir à la musique aléatoire, aux happenings, 
à cette multidisciplinarité artistique qui laisse jaillir la spontanéité de l'interprétation. Aussi cette traduction est-elle insuffisante, sinon erronée : si l'on ne peut pas penser l'actuel sans l'inclure dans une pensée sur le temps ( «c'est le présent qui passe, qui définit l'actuel ${ }^{24}$ »), l'actuel réfère également à ce qui existe «en acte", à ce qui est «action »; l'étymologie latine de ces termes provient d'ailleurs de la même racine : «acte» vient d'acta, pluriel d'actum qui est le participe passé substantivé d'agere, "agir». "Action » découle d'actio et dérive d'actum, supin d'agere. "Actuel» dérive d'actualis, «actif, agissant », lui-même venant d'actum, forme nominale du verbe agere. L'actuel impose donc deux champs de compréhension : l'agir d'un côté; de l'autre un rapport au temps. Cette tension se situe d'ailleurs à la base même du geste automatiste de Borduas qui influença Mercure, notamment dans $\mathrm{H}_{2} \mathrm{O}$ per Severino (1965) 25. Or, l'esthétique musicale des actualistes puise à la source de l'automatisme surrationnel conçu comme «source intarissable qui coule sans entrave de l'homme ${ }^{26}$ ». De la peinture automatiste à la musique actuelle ${ }^{27}$, «la conséquence est plus importante que le but ${ }^{28}$ » : il s'agit de «chercher sans vouloir trouver, sans but, sans finalité [...] ici et maintenant; l'important est le chemin ${ }^{29}$ ». Inventer, explorer sans cesse en s'aventurant loin des schèmes - d'où le concept d'« exploréen » de Claude Gauvreau, qui évoque l'idée de la création conçue comme un voyage en son for intérieur, dans un rapport étroit à l'autre.

\section{Référents psychosociaux}

Toute identité est un rapport dialectique entre l'individu et la société. Elle se construit en fonction de pratiques collectives partagées dans lesquelles on va pouvoir se découvrir des valeurs communes. Ces valeurs laissent penser que nous ne sommes pas seuls au monde, mais que l'on partage, ensemble, des pratiques et des visées similaires. On peut, à cet effet, rappeler quelques-unes des déclarations des actualistes se remémorant leurs débuts : «[...] à travers les artistes publiés par Recommended Records et les compagnies qui distribuaient ces disques un peu partout, nous avons trouvé une famille $3^{\circ}$ », ou encore : «[...] nous avons été capables de nous identifier à un mouvement ${ }^{31}$ ", «[...] notre musique s'insérait dans un courant, que d'autres personnes faisaient, un peu partout, une musique aussi "indéfinissable" que la nôtre ${ }^{32}$ ».

Qu'est-ce qui unifie musique actuelle et réalité ambiante? En tenant compte des événements sociaux qui ont bouleversé le Québec et des témoignages des musiciens (notamment celui de Hétu qui suppose «un grand lien entre le Québec et la musique actuelle» - mais peut-être moins, précise-t-elle, «entre la culture québécoise et la musique actuelle 33 »), en quoi
24. Deleuze, [1977]1996, p. 183.

25. Pour ce qui est des liens entre Mercure et l'automatisme québécois, voir "Pierre Mercure fait-il de l'automatisme en musique?", conférence de Lyse Richer-Lortie, 1976/02/04. Communiqué de presse o6-M, Pgo/1-19.4. Gouvernement du Québec, Ministère des Affaires culturelles, Musée d'Art Contemporain (Montréal). Original dactylographié, 4 pages. Fonds Pierre Mercure, Bibliothèque et Archives nationales du Québec.

26. Borduas, 1987, p. 413.

27. Voir Stévance, 2011.

28. Borduas, 1987, p. 447.

29. Léandre, 2008, p. 93.

30. Danielle Palardy Roger citée in Beaucage, 2004, p. 42.

31. Danielle Palardy Roger, in Marin, 2005. www.radiocanada.ca/ culture/modeledocument.asp?sect ion $=$ musique\&prov=reportage\&id Region $=1 \&$ idEntite $=3045$.

32. Joane Hétu, citée in Beaucage, 2004 , p. 42.

33. Joane Hétu, citée in Arroyas, 2005 , p. 8. 
la musique actuelle est-elle "québécoise »? Si l'on retient par l'adjectif «québécois(e)» les significations du Petit Robert (2009), on note que le terme désigne l'originaire de la ville ou de la province du Québec, et la langue française telle qu'elle est parlée et écrite au Québec. Ces deux idées réfèrent à ce qui est propre au Québec relativement à son territoire, à sa civilisation, à sa culture. Étant donné ces références (culturelle, géographique et linguistique), serait dite «québécoise » toute musique composée au Québec par un ou des créateurs québécois dont la démarche tient compte de la tradition et de l'évolution historique particulière de sa nation d'appartenance (ou de l'idée qu'il s'en fait), faisant ainsi de ladite œuvre une spécificité culturelle. Mais : "Qualifier une musique de québécoise, c'est aussi faire intervenir des stratégies cognitives complexes où se mêlent l'identification de traits culturels propres aux gens, à la langue ou au pays, et l'expression plus ou moins émotive, plus ou moins rationnelle, d'aspirations sociopolitiques 34 ». Il faut alors établir sinon les attaches, du moins les relations entre l'univers sonore des actualistes et les valeurs propres au territoire dans lequel tous se sont réunis pour forger leur style musical.

Les bouleversements sociaux ont, dès les années 1960, exhorté les Québécois à reconnaître leur identité ethnique par l'exploration de leur culture et l'affirmation de leur langue. Les actualistes ont tôt participé à cette prise de conscience d'une identité nationale : ils révèlent, au même titre que la chanson, la culture québécoise qu'ils vivent et chantent pleinement tout en pointant, comme d'ailleurs les premiers chansonniers, le danger d'américanisation du peuple québécois. C'est aussi pourquoi ils ont développé leurs propres sociétés de production, telles que l'Ensemble de musique improvisée de Montréal, Nébu ou Les Granules. Les festivals de jazz et de musique improvisée se développent de plus en plus sur l'ensemble du territoire québécois. Se dessinent alors les orientations de ce qui définira la musique actuelle : une imagination débridée, une création collective et chaleureuse, une création musicale qui s'active en présence d'un public averti et engageant, une musique au pluriel dont le sens n'est jamais déterminé d'avance, un territoire sonore franchissant les barrières entre le savant et le populaire et exprimant de nouvelles valeurs de sociabilité pour s'éloigner des lois et du marché de l'art.

\section{Conclusion}

La musique actuelle s'inscrit et participe de l'histoire de la société québécoise : elle témoigne de son émergence et des événements socioculturels qui l'ont bouleversée, ainsi que de sa recherche identitaire à travers la quête de 
sa spécificité musicale. On ne saurait alors considérer qu'elle «n'est pas un reflet direct du Québec ${ }^{35}$ ", notamment lorsqu'elle est en rapport avec des conceptions artistiques affichées par d'autres courants culturels qui ont marqué la Nation depuis le Refus Global, sans oublier la Semaine internationale de musique actuelle, événement grâce auquel Mercure a pu ouvrir - et même inscrire - la culture québécoise aux recherches artistiques les plus expérimentales. Or, le courant de la musique actuelle a puisé ses stratégies identitaires à même les principes esthétiques et sociaux qui motivaient la SIMA en 1961, et il s'agit bien d'une déconstruction des critères esthétiques normatifs pour montrer que «L'art est un devoir de subversion, de bouleversement, de réflexion. C'est une célébration de la vie. L'artiste est dérangeant ${ }^{36}{ }^{\star}$. Pour Derrida, le but de la déconstruction vise à défaire par l'analyse ce qui a été structuré, c'est-à-dire l'ensemble des structures qui forment l'élément discursif (culture, références, etc.). La musique actuelle se positionne contre ce qui sous-tend les oppositions conceptuelles binaires inhérentes au système de pensée de l'histoire de la musique. Les actualistes cherchent à affirmer un autre sens possible à la création musicale en déconstruisant ses fondements à partir de ce qui a été occulté (la création collective et spontanée, les lieux de production, les conditions d'écoute, etc.) afin de faire émerger un art inclassable car «androgyne», «métissé», contenant des pôles auparavant opposés.

L'expression musique actuelle a donc été utilisée de deux façons : 1) le sens que lui donnait Pierre Mercure (musique expérimentale à l'image de l'américanité ambiante, et musique de notre temps); 2) le sens que lui ont donné les membres fondateurs du mouvement dans les années 1980 (une musique hybride avec une esthétique définie, à l'image de l'époque; une musique reprenant ce que les institutions ont rejeté). Depuis 1990, le terme s'est stabilisé et désigne en propre les Productions SuperMusique. Entre les deux, on doit compter sur ce long processus d'évolution que la chronologie tracée en annexe 1 a souhaité illustrer. Raymond Gervais 37 mentionne cette distinction entre deux époques, mais il est davantage question ici d'un processus de maturation d'une expression qui deviendra un courant musical dès les années 1980. La SIMA de Pierre Mercure a été un déclencheur de cette effervescence qu'a connue la création musicale québécoise à partir des années 1960. Mais, ainsi que nous le précise Marie-Thérèse Lefebvre, «c'est Mercure-réalisateur plutôt que Mercure-compositeur» qui en est à l'origine : ce n'est pas ses compositions que l'on retient dans la constitution de la musique actuelle au Québec. Aucune de ses œuvres ne constitue un point de repère - mais c'est son festival, son esprit et sa programmation $3^{8}$.
35. Joane Hétu, citée in Arroyas, 2005 , p. 8.

36. Léandre, 2008, p. 118-119.

37. Gervais, 1990.

38. Je remercie Serge Lacasse de m’avoir encouragée à écrire cet article, ainsi que Marie-Thérèse Lefebvre, Danielle Palardy Roger, Joane Hétu et Jean Derome pour leurs précieuses informations, le temps qu'elles et il ont bien voulu consacrer à répondre à mes questions. 


\section{B I B L I O G RAP H IE}

Arroyas, Frédérique (2005), «Entretien avec Joane Hétu», Critical Studies in Improvisation / Études critiques en improvisation, vol. 1, $\mathrm{n}^{\circ}$ 2, p. 4-9.

Beaucage, Réjean (2004), «25 ans de SuperMusique. Contre vents et marées! », La Scena Musicale, vol. 10, $\mathrm{n}^{\circ} 1$, septembre, p. 42

Borduas, Paul-Émile (1987), «Projections libérantes », in Écrits 1, André-G. Bourassa, Jean Fisette et Gilles Lapointe (dir.), Montréal, PUM, p. 413.

Bourdieu, Pierre et Wacquant, Loïc (1992), Réponses. Pour une anthropologie réflexive, Paris, Seuil.

CAGE, John (1961), Silence, Lectures and Writings by John Cage, Middletown, Wesleyan University Press, 1961.

CôtÉ, Michel F. (1995), «Cirque nominatif», Circuit, vol. 6, n² 2, p. 27-29.

Deleuze, Gilles ([1977] 1996), «L'actuel et le virtuel », in Dialogues avec Claire Parmet, Paris, Flammarion, p. 183

Gervais, Raymond (1990), «Montréal, Musiques actuelles, 1960-1990», programme officiel du Festival Montréal musiques actuelles/New Music America, p. 7.

LÉANDre, Joëlle (2008), À voix basse. Entretiens avec Franck Médioni, Paris, MF.

Lefebvre, Marie-Thérèse (1992), «L'influence de John Cage au Québec : résistances et convergences », Les Cahiers de L'ARMuQ, n ${ }^{\circ}$, mai, p. 87-107

Lefebvre, Marie-Thérèse et PInson, Jean-Pierre (2009), Chronologie musicale du Québec, 1535 2004, Québec, Septentrion.

MARIN, Lili (2005), «La musique actuelle peut-elle vieillir?», Radio-Canada: Guide Culturel, 28 mars [www.radio-canada.ca/culture/modeledocument.asp?section=musique \&prov=repor tage \&id\%2oRegion=1\&idEntite=3045].

MClean, Eric (1961), «One Narrow Aspect of Today’s Music», The Montreal Star, 12 août, p. 10.

NATtiEZ, Jean-Jacques (1999), «Y a-t-il une musique québécoise? », La Musique, la recherche et la vie. Un dialogue et quelques dérives, Montréal, Leméac, p. 206.

PÉpin, Clermont (1961), «Montréal : la Semaine internationale de musique actuelle», The Canadian Music Journal, vol. 6, automne, p. 29-31.

Raymond, Dyane (2007), Jean Derome, L’homme musique, Montréal,Varia.

RIVEST, Johanne (1997), «Atlas Eclipticalis, commande montréalaise», Circuit, «Québécage», vol. $8, n^{\circ} 2$, p. 61-69.

Rivest, Johanne (1998), «La représentation des avant-gardes à la Semaine internationale de musique actuelle (Montréal, 1961)», RMUC, 19/1, 1998, p. 50-68.

StÉvance, Sophie (2011), Musique actuelle, livre à paraître aux PUM.

VAllerand Jean (1961), «Le Festival de musique actuelle», Le Devoir, 14 août, p. 8. 